PROCEEDINGS OF THE

AMERICAN MATHEMATICAL SOCIETY

Volume 129, Number 4, Pages 965-973

S 0002-9939(00)05055-3

Article electronically published on October 19, 2000

\title{
DESCENT ALGEBRAS, HYPERPLANE ARRANGEMENTS, AND SHUFFLING CARDS
}

\author{
JASON FULMAN
}

(Communicated by John R. Stembridge)

\begin{abstract}
Two notions of riffle shuffling on finite Coxeter groups are given: one using Solomon's descent algebra and another using random walk on chambers of hyperplane arrangements. These coincide for types $A, B, C, H_{3}$, and rank two groups but not always. Both notions have the same simple eigenvalues. The hyperplane definition is especially natural and satisfies a positivity property when $W$ is crystallographic and the relevant parameter is a good prime. The hyperplane viewpoint suggests deep connections with Lie theory and leads to a notion of riffle shuffling for arbitrary real hyperplane arrangements and oriented matroids.
\end{abstract}

\section{INTRODUCTION AND BACKGROUND}

Using ideas from $[\mathrm{BaD},[\mathrm{BB}$, and particularly $[\mathrm{BBHT}]$, we give a definition of card-shuffling measures $M_{W, x}$ on finite Coxeter groups. Here $x \neq 0$ is a real number, and $M_{W, x}$ satisfies the measure property $\sum_{w \in W} M_{W, x}(w)=1$. In general these measures will be signed, i.e. it is possible that $M_{W, x}(w)<0$ for some element $w$. The measures $M_{W, x}$ convolve and have nice eigenvalues.

The measures $M_{W, x}$ have received considerable attention in the cases that $W$ is of type $A$ or $B$. The type $A$ case appeared in $[\mathrm{BaD}$ in the theory of riffle shuffling. Results for biased shuffles appear in [F3]. Type $B$ riffle shuffles are considered in [BB]. As is evident from [BB] and [Ha], $M_{A_{n}, x}$ and $M_{B_{n}, x}$ are related to the Poincaré-Birkhoff-Witt theorem and to splittings of Hochschild homology. Section 3.8 of [SS] describes the measures $M_{A_{n}, x}$ in the language of Hopf algebras. Stanley St] connects riffle shuffling with representation theory of the symmetric group, recasting work of $[\mathrm{BaD}],[\mathrm{F} 3$ and giving a new approach to recent work in the random matrix community. The paper [F5] uses representation theory to analyze riffle-shuffles followed by cuts.

We then give a second definition $H_{W, x}$ of riffle shuffling for finite Coxeter groups using work of Bidigare, Hanlon, and Rockmore [BHR]. They describe how putting non-negative weights summing to one on the faces of a hyperplane arrangement induces a random walk on its chambers. This procedure will be recalled in Section 3 Brown and Diaconis $\mathrm{BrD}$ generalize these chamber walks to oriented matroids and give many examples. Bidigare $\mathrm{B}$ realized type $A$ shuffling as a special case, with face weights as binomial coefficients. We give a definition for all finite Coxeter

Received by the editors January 30, 1998 and, in revised form, May 18, 1998 and July 15, 1999.

1991 Mathematics Subject Classification. Primary 20F55, 20 P05.

(C)2000 American Mathematical Society 
groups using group theoretic weights. As a by-product, it will be seen that if $W$ is crystallographic and $p$ is a good prime, then $M_{W, p}(w) \geq 0$ for all $w \in W$. It will emerge that the eigenvalues and multiplicities are the same as for the descent algebra definition. The weights in the construction of $H_{W, x}$ are then expressed in a completely combinatorial way, yielding a definition for arbitrary real hyperplane arrangements.

The follow-up work [F1], F2 connects the measures $H_{W, x}$ with the semisimple orbits of the adjoint action of a finite group of Lie type on its Lie algebra. One long term goal is to naturally associate to a semisimple conjugacy class or adjoint orbit an element $w$ of the Weyl group, refining the well-known map to conjugacy classes of $W$. This was partially realized in [F1], [F4].

\section{Descent Algebra Definition of ShufFling FOR COXETER GROUpS}

Let $W$ be a finite Coxeter group with $\Pi$ a base of fundamental roots. For $w \in W$, let $\operatorname{Des}(w)$ be the set of simple positive roots mapped to negative roots by $w$ (also called the descent set of $w)$. For $J \subseteq \Pi$, let $X_{J}=\{w \in W \mid \operatorname{Des}(w) \cap J=\emptyset\}$ and $x_{J}=\sum_{w \in X_{J}} w$. Let $\lambda$ be an equivalence class of subsets of $\Pi$ under the equivalence relation $J \sim K$ whenever $w(J)=K$ for some $w \in W$. Let $\lambda(K)$ denote the equivalence class of $K$. For $K \subseteq J \subseteq \Pi$ define $\mu_{K}^{J}=\frac{\left|\left\{w \in X_{J}: w(K) \subseteq \Pi\right\}\right|}{|\lambda(K)|}$. Set $\mu_{K}^{J}=0$ if $K \nsubseteq J$. Let $\left(\beta_{K}^{J}\right)$ be the matrix inverse of $\left(\mu_{K}^{J}\right)$. Define $e_{\lambda}$ in the descent algebra of $W$ by

$$
e_{\lambda}=\sum_{J \in \lambda} \frac{\sum_{K \subseteq J} \beta_{K}^{J} x_{K}}{|\lambda|} .
$$

These $e_{\lambda}$ are orthogonal idempotents which sum to the identity element of $W$ BBHT. Let $\|\lambda\|$ denote $|J|$ for any $J$ in the equivalence class $\lambda$.

Definition 1. For $W$ a finite Coxeter group and $x \neq 0$, define a signed probability measure $M_{W, x}$ on $W$ by

$$
M_{W, x}=\sum_{\lambda} \frac{e_{\lambda}}{x^{\|\lambda\|}} .
$$

For $w \in W$, let $M_{W, x}(w)$ be the coefficient of $w$ in $M_{W, x}$.

Since the $e_{\lambda}$ are orthogonal idempotents, $M_{W, x}$ satisfies the following convolution property in the group algebra of $W$ :

$$
M_{W, x} M_{W, y}=M_{W, x y} .
$$

Proposition 1. $M_{W, x}$ is a signed probability measure on $W$.

Proof. Writing each $e_{\lambda}$ as $\sum_{w \in W} c_{\lambda}(w) w$ it must be proved that

$$
\sum_{w, \lambda} \frac{c_{\lambda}(w)}{x^{\|\lambda\|}}=1
$$

This follows from the stronger assertion that $\sum_{w} c_{\lambda}(w)$ is 0 if $\|\lambda\|>0$ and is 1 if $\|\lambda\|=0$. Since $\beta_{\emptyset}^{\emptyset}=\frac{1}{|W|}$, it follows by definition that $e_{\emptyset}=\frac{\sum_{w \in W} w}{|W|}$. Thus $\sum_{w} c_{\lambda}(w)=1$ if $\|\lambda\|=0$. Since the $e_{\lambda}$ are idempotents, the value of $\sum_{w} c_{\lambda}(w)$ is either 0 or 1 . Since $\sum_{\lambda} e_{\lambda}=1$, clearly $\sum_{w, \lambda} c_{\lambda}(w)=1$. Combining this with the fact that $\sum_{w} c_{\lambda}(w)=1$ if $\|\lambda\|=0$ shows that $\sum_{w} c_{\lambda}(w)=0$ if $\|\lambda\|>0$.

Proposition 2 illustrates the definition by computing $M_{G_{2}, x}$. 


\section{Proposition 2.}

$$
M_{G_{2}, x}(w)= \begin{cases}\frac{(x+5)(x+1)}{12 x^{2}} & \text { if } d(w)=0, \\ \frac{(x+1)(x-1)}{12 x^{2}} & \text { if } d(w)=1, \\ \frac{(x-1)(x-5)}{12 x^{2}} & \text { if } d(w)=2 .\end{cases}
$$

Proof. Letting $V$ be the hyperplane in $R^{3}$ consisting of vectors whose coordinates add to 0 , it is well known that a root system consists of $\pm\left(\varepsilon_{i}-\varepsilon_{j}\right)$ for $i<j$ and $\pm\left(2 \varepsilon_{i}-\varepsilon_{j}-\varepsilon_{k}\right)$ where $\{i, j, k\}=\{1,2,3\}$. Let $A=\varepsilon_{1}-\varepsilon_{2}$ and $B=-2 \varepsilon_{1}+\varepsilon_{2}+\varepsilon_{3}$ be a base of positive simple roots. All equivalence classes $\lambda$ of subsets of $\Pi$ have size one. Some computation gives

$$
\begin{aligned}
e_{\emptyset} & =\frac{1}{12} x_{\emptyset} \\
e_{A} & =-\frac{1}{4} x_{\emptyset}+\frac{1}{2} x_{A}, \\
e_{B} & =-\frac{1}{4} x_{\emptyset}+\frac{1}{2} x_{B}, \\
e_{A, B} & =\frac{5}{12} x_{\emptyset}-\frac{1}{2} x_{A}-\frac{1}{2} x_{B}+x_{A, B},
\end{aligned}
$$

from which the result follows.

Left multiplication in the group algebra of $W$ by $M_{W, x}$ can be thought of as performing a signed random walk on $W$. The corresponding transition matrix is a $|W|$ by $|W|$ matrix. Proposition 3 determines its eigenvalues and evaluates $M_{W, x}(w)$ when $w$ is the identity or longest element. It was known previously for types $A$ and $B$ (part 1 in $[\mathrm{Ha}]$, the rest in $[\mathrm{BaD}]$ and $[\mathrm{BB}]$ ).

Proposition 3. Let $W$ be a finite Coxeter group of rank $n$. Let id be the identity element of $W$ and $w_{0}$ the longest element of $W$. Let $m_{1}, \cdots, m_{n}$ be the exponents of $W$.

(1) The eigenvalues of $M_{W, x}$ are $\frac{1}{x^{i}}$ for $0 \leq i \leq n-1$ with multiplicity the number of $w \in W$ with fixed space of dimension $n-i$.

(2) $M_{W, x}(i d)=\frac{\prod_{i=1}^{n}\left(x+m_{i}\right)}{x^{n}|W|}$.

(3) $M_{W, x}\left(w_{0}\right)=\frac{\prod_{i=1}^{n}\left(x-m_{i}\right)}{x^{n}|W|}$.

Proof. The first claim is easily reduced to Theorem 7.15 of [BBHT]. For the second claim note from the first that summing over $\lambda$ with $\|\lambda\|=i$ the coefficient of the identity in $e_{\lambda}$ gives $\frac{1}{|W|}$ times the number of $w$ with fixed space of dimension $n-i$. Thus

$$
M_{W, x}(i d)=\frac{1}{x^{n}|W|} \sum_{w \in W} x^{\operatorname{dim}(f i x(w))}=\frac{\prod_{i=1}^{n}\left(x+m_{i}\right)}{x^{n}|W|},
$$

the second equality being an identity of Shephard and Todd $\underline{\mathrm{ST}}$. The third claim follows analogously to the second, but in the proof of Theorem 7.15 of [BBHT] one takes inner products with the alternating representation instead of with the trivial representation. 


\section{Hyperplane WALK Definition OF SHUfFling FOR Coxeter GROUPS}

First it is necessary to review the paper BHR. Let $\mathcal{A}=\left\{H_{i}: i \in I\right\}$ be a central hyperplane arrangement (i.e. $\bigcap_{i \in I} H_{i}=0$ ) for a real vector space $V$. Let $\gamma$ be a vector in the complement of $\mathcal{A}$. Every $H_{i}$ partitions $V$ into three pieces: $H_{i}^{0}=H_{i}$, the open half space $H_{i}^{+}$of $V$ containing $\gamma$, and the open half space $H_{i}^{-}$ of $V$ not containing $\gamma$. The faces of $\mathcal{A}$ are defined as the non-empty intersections of the form $\bigcap_{i \in I} H_{i}^{\epsilon_{i}}$, where $\epsilon_{i} \in\{0,-,+\}$. Equivalently, $\mathcal{A}$ cuts $V$ into regions called chambers and the faces are the faces of these chambers viewed as polyhedra. A random process on chambers (henceforth called the BHR walk) is then defined as follows. Assign weights $v(F)$ to the faces of $\mathcal{A}$ in such a way that $v(F) \geq 0$ for all $F$ and $\sum_{F} v(F)=1$. Pick a starting chamber $C_{0}$. At step $i$, pick a face $F_{i}$ with the chance that $F_{i}=F$ equal to $v(F)$ and define $C_{i}$ to be the unique chamber whose closure contains $F_{i}$ and which among such chambers is separated from $C_{i-1}$ by the fewest number of hyperplanes.

For the remainder of this section, $\mathcal{A}$ will be the arrangement of root hyperplanes for a finite Coxeter group $W$.

Proposition $4([\overline{\mathrm{H}}])$. The chambers of $\mathcal{A}$ correspond to the elements of $W$. The faces of $\mathcal{A}$ correspond to left cosets of parabolic subgroups of $W$. The faces contained in the closure of $w$ are the left cosets $w W_{J}$.

The next lemma will be of use.

Lemma 1. Let $C_{0}$ be the chamber of $\mathcal{A}$ corresponding to the identity. Let $v(F)$ be the weight on a face $F$ in a BHR walk on a finite Coxeter group. Then the chance that the chamber $C_{1}$ corresponds to $w$ is equal to

$$
\sum_{K \subset \Pi-\operatorname{Des}(w)} v\left(w W_{K}\right)
$$

Proof. The chance that $C_{1}$ corresponds to $w$ is equal to $\sum_{F} v(F)$, where the sum is over all faces $F$ in the closure of $w$ and such that $w$ is the chamber whose closure contains $F$ and which is closest to the identity. The faces in the closure of $w$ are the cosets $w W_{K}$, for $K$ an arbitrary subset of $\Pi$. The chambers whose closure contains the face $w W_{K}$ are the elements of the coset $w W_{K}$. Proposition 1.10 of [H] shows that $w$ is the unique shortest element in the coset $w W_{K}$ precisely when $K \subset \Pi-\operatorname{Des}(w)$.

Let $L$ be the set of intersections of the hyperplanes in $\mathcal{A}$, taking $V \in L$. (This lattice is not the same as the face lattice.) Partially order $L$ by reverse inclusion. Recall that the Moebius function $\mu$ is defined by $\mu(X, X)=1$ and $\sum_{X<Z<Y} \mu(Z, Y)=0$ if $X<Y$ and $\mu(X, Y)=0$ otherwise. The characteristic polynomial of $L$ is defined as

$$
\chi(L, x)=\sum_{X \in L} \mu(V, X) x^{\operatorname{dim}(X)} .
$$

(This is not the standard definition in which the exponent of $x$ is $n-\operatorname{rank}(X)$. This distinction is important since the definitions do not agree for subposets.) For $J \subseteq \Pi$, let Fix $\left(W_{J}\right)$ denote the fixed space of the parabolic subgroup $W_{J}$ in its action on $V$. Let $L^{F i x\left(W_{J}\right)}$ be the subposet $\left\{Y \in L \mid Y \geq F i x\left(W_{J}\right)\right\}$. Finally, $N_{G_{1}}\left(G_{2}\right)$ denotes the normalizer of the group $G_{2}$ in $G_{1}$. 
Definition 2. Let $W$ be a finite Coxeter group of rank $n$. Define $H_{W, x}(w)$ by the formula

$$
H_{W, x}(w)=\sum_{K \subseteq \Pi-D e s(w)} \frac{\left|W_{K}\right| \chi\left(L^{F i x\left(W_{K}\right)}, x\right)}{x^{n}\left|N_{W}\left(W_{K}\right)\right||\lambda(K)|} .
$$

Let $W$ be a finite irreducible crystallographic Coxeter group. Recall that $p$ is said to be a bad prime if $p$ divides the coefficient of some root of $W$ when expressed as a linear combination of simple roots. Alternatively, $p$ is a bad prime if it is less than the maximum exponent of $W$ but not equal to an exponent of $W$. A prime $p$ is said to be good if $p$ is not bad.

Proposition 5. Let $W$ be a finite irreducible crystallographic Coxeter group of rank $n$. Then if $p$ is a good prime for $W$, the measure $H_{W, p}$ can be viewed as a special instance of the BHR walks with face weights

$$
v_{p}\left(w W_{K}\right)=\frac{\left|W_{K}\right| \chi\left(L^{F i x\left(W_{K}\right)}, p\right)}{p^{n}\left|N_{W}\left(W_{K}\right)\right||\lambda(K)|} .
$$

In particular, $H_{W, p}(w) \geq 0$ for all $w \in W$.

Proof. It will be proved in Section 4 that the face weights sum to 1. By Lemma 1 it is sufficient to show that $\chi\left(L^{F i x\left(W_{K}\right)}, p\right)$ is non-negative for $p$ a good prime. One of the main results of Orlik and Solomon $\mathrm{OS}$ is the factorization

$$
\chi\left(L^{F i x\left(W_{K}\right)}, x\right)=\prod_{i=1}^{\operatorname{dim}\left(\operatorname{Fix}\left(W_{K}\right)\right)}\left(x-b_{i}^{K}\right),
$$

where the $b_{i}^{K}$ are positive integers. From the tables in their paper, the $b_{i}^{K}$ are all less than or equal to the maximum exponent of $W$.

Remarks. 1. The BHR process can still be considered with negative face weights, viewed as a transition matrix with possibly negative entries. Using the face weights of Proposition 5 where $W$ is crystallographic and $x$ is a bad prime gives a first natural collection of examples where some of the face weights are negative. As will emerge from Section 4 the eigenvalues of the transition matrix are positive. We also note that the $W$-invariance of the weights implies that the process can be viewed as left multiplication by an element of the group algebra. It would be interesting to prove that a cut-off phenomenon occurs for these chains in the sense of [BaD].

2. Although the expression $H_{W, x}(w)$, viewed as a function of $x$ for fixed $w$, factors into linear terms when $W$ is of type $A, B$ or $I$, this property does not hold in general (one simple counterexample is taking $W$ of type $H_{4}$ ). However the proof of Corollary 5 implies that the face weights $v_{x}\left(w W_{K}\right)$ do factor into linear terms as a function of $x$. This shows the naturality of the hyperplane viewpoint. We observe (though it is not clear from the definition) that in type $A$ these weights agree with the binomial coefficients weights in BHR.

3. The values $|\lambda(K)|$ have been tabulated $[\mathrm{C} 2]$.

\section{Riffle Shuffling FOR REAL HYPERPlane ARRAngEMENTS}

This section defines riffle shuffling for arbitrary real hyperplane arrangements and explores some of its properties. As a corollary it will be shown that $M_{W, x}$ and $H_{W, x}$ agree for many types. In fact the main definition of this section extends to 
oriented matroids by replacing the terms "chamber" and "hyperplane intersection" by the terms "tope" and "flat". For clarity of exposition we suppose that $\mathcal{A}$ is a real hyperplane arrangement, possibly non-central, with a finite number of hyperplanes. Brown and Diaconis [BrD] verified that the BHR walks extend to this setting.

Recall the zero map from the face lattice to the intersection lattice. This map $z$ sends a face $\bigcap_{i \in I} H_{i}^{\epsilon_{i}}, \epsilon_{i} \in\{0,-,+\}$ to $\bigcap_{i \in I: \epsilon_{i}=0} H_{i}$. Geometrically, $z$ maps a face $F$ to its support, namely the intersection of $V$ with all hyperplanes containing $F$.

Lemma $2([\mathrm{Z}])$. For all $Y \in L$,

$$
\begin{aligned}
\left|z^{-1}(Y)\right| & =\sum_{Z \geq Y}|\mu(Y, Z)|=\sum_{Z \geq Y}(-1)^{\operatorname{dim}(Y)-\operatorname{dim}(Z)} \mu(Y, Z) \\
& =(-1)^{\operatorname{dim}(Y)} \chi\left(L^{Y},-1\right) .
\end{aligned}
$$

Lemma 3 ([OS]). For all $X \in L$,

$$
\sum_{\substack{Y \in L \\ Y \geq X}} \chi\left(L^{Y}, x\right)=x^{\operatorname{dim}(X)}
$$

Proof.

$$
\begin{aligned}
\sum_{\substack{Y \in L \\
Y \geq X}} \chi\left(L^{Y}, x\right) & =\sum_{Y \geq X} \sum_{Z \geq Y} \mu(Y, Z) x^{\operatorname{dim}(Z)} \\
& =\sum_{Z \in L} \sum_{Y: X \leq Y \leq Z} \mu(Y, Z) x^{\operatorname{dim}(Z)} \\
& =x^{\operatorname{dim}(X)} .
\end{aligned}
$$

Definition 3. Define a one-parameter family of card-shuffling walks on the chambers of a real hyperplane arrangement $\mathcal{A}$ in $n$ dimensions as the BHR walks with face weights

$$
v_{x}(F)=\frac{\chi\left(L^{z(F)}, x\right)}{x^{n}\left|z^{-1}(z(F))\right|}=(-1)^{\operatorname{dim}(z(F))} \frac{\chi\left(L^{z(F)}, x\right)}{x^{n} \chi\left(L^{z(F)},-1\right)} .
$$

Lemmas 2 and 3 imply that these face weights sum to one:

$$
\sum_{F} v_{x}(F)=\sum_{\substack{Y \in L \\ Y \geq V}} \sum_{F: z(F)=Y} \frac{\chi\left(L^{z(F)}, x\right)}{x^{n}\left|z^{-1}(z(F))\right|}=\sum_{\substack{Y \in L \\ Y \geq V}} \frac{\chi\left(L^{Y}, x\right)}{x^{n}}=1 .
$$

The $v_{x}(F)$ are not necessarily positive, in which case the BHR walks take the extended meaning in the remark after Proposition 5. To show that Definition 3 extends Definition 2, the following lemma will be helpful.

Lemma 4 ([OS]).

$$
\frac{\left|N_{W}\left(W_{K}\right)\right||\lambda(K)|}{\left|W_{K}\right|}=(-1)^{\operatorname{dim}\left(z\left(w W_{K}\right)\right)} \chi\left(L^{z\left(w W_{K}\right)},-1\right) .
$$

We remark that combining the fact that the face weights sum to one with Lemma 4 yields the intriquing identity

$$
\sum_{K \subseteq \Pi}(-1)^{n-|K|} \frac{|W|}{\left|W_{K}\right|} \frac{\chi\left(L^{F i x\left(W_{K}\right)}, x\right)}{\chi\left(L^{F i x\left(W_{K}\right)},-1\right)}=x^{n} .
$$


Setting $x=-1$ in this identity gives the alternating sum formula

$$
\sum_{K \subseteq \Pi}(-1)^{|K|} \frac{|W|}{\left|W_{K}\right|}=1
$$

which has a topological proof [So1] as well as applications in the invariant theory of Coxeter groups $[\mathrm{H}]$. Generalizations and $q$-analogs related to the Steinberg character appear in Section 6.2 of $\mathrm{C} 2$.

Theorem 1. The measures $H_{W, x}$ arise from Definition 3 with $\mathcal{A}$ equal to the arrangement of root hyperplanes of $W$.

Proof. The theorem follows by showing that the face weights of Definition 2 are equal to the weights Definition 3 associates to the arrangement of root hyperplanes of $W$. Thus it is necessary to prove that

$$
\frac{\left|W_{K}\right| \chi\left(L^{F i x\left(W_{K}\right)}, x\right)}{x^{n}\left|N_{W}\left(W_{K}\right)\right||\lambda(K)|}=(-1)^{\operatorname{dim}\left(z\left(w W_{K}\right)\right)} \frac{\chi\left(L^{z\left(w W_{K}\right)}, x\right)}{x^{n} \chi\left(L^{z\left(w W_{K}\right)},-1\right)} .
$$

Since $z\left(W_{K}\right)=F i x\left(W_{K}\right), L^{z\left(w W_{K}\right)}$ is isomorphic to $L^{F i x\left(W_{K}\right)}$, and thus the result follows from Lemma t.

Theorem 1 shows that the measures $H_{W, x}$ can be easily computed from the tables of Orlik and Solomon. For example, one can check that $H_{W, x}$ agrees with the formula for $M_{W, x}$ on the identity and longest element of $W$ (see the follow-up [F1] for a more conceptual proof). The point of including Definition 2 was to show that the face weights can be expressed group theoretically.

Theorem 2. The Markov chain associated to the hyperplane arrangement $\mathcal{A}$ by Definition 1 has eigenvalues $\frac{1}{x^{2}}$ with multiplicity $\sum_{X \in L: \operatorname{dim}(X)=n-i}|\mu(V, X)|$ for $0 \leq i \leq n-1$. In particular, the eigenvalues and multiplicities of $H_{W, x}$ agree with those of $M_{W, x}$.

Proof. For the first assertion, $[\mathrm{BrD}]$ (extending the paper $[\mathrm{BHR}]$ to the affine case) proves that the eigenvalues for their chamber walks are indexed by elements $X \in L$ and are equal to $\sum_{F: z(F) \geq X} v(F)$ with multiplicity $|\mu(V, X)|$. Definition 3 and Lemma 3 imply that

$$
\sum_{F: z(F) \geq X} v_{x}(F)=\frac{1}{x^{n}} \sum_{Y \geq X} \chi\left(L^{Y}, x\right)=\frac{1}{x^{n-\operatorname{dim}(X)}} .
$$

The second assertion follows from the factorization of the characteristic polynomial of a root arrangement $\chi(L, x)=\prod_{i}\left(x-m_{i}\right)$ together with the result of Shephard and Todd used in part 2 of Proposition 3 ,

From Theorem 2 two natural questions emerge. First, do $H_{W, x}$ and $M_{W, x}$ induce the same measure on conjugacy classes? Second, recall that the Fourier transform $\widehat{P}(\rho)$ of a probability distribution $P$ at a representation $\rho$ is $\sum_{w \in W} P(w) \rho(w)$. Do these matrices have the same eigenvalues for $H_{W, x}$ and $M_{W, x}$ for all irreducible representations?

Theorem 3 shows that in many cases $H_{W, x}$ and $M_{W, x}$ agree.

Theorem 3. Let $W$ be a finite irreducible Coxeter group of type $A, B, C, H_{3}$ or rank 2. Then $H_{W, x}=M_{W, x}$. 
Proof. The rank 2 cases are straightforward. For the case of $H_{3}$, letting $d(w)$ be the number of descents of $w$ it follows from computations in B3 that

$$
M_{H_{3}, x}(w)= \begin{cases}\frac{(x+9)(x+5)(x+1)}{120 x^{3}} & \text { if } d(w)=0, \\ \frac{(x+5)(x+1)(x-1)}{120 x^{3}} & \text { if } d(w)=1, \\ \frac{(x+1)(x-1)(x-5)}{120 x^{3}} & \text { if } d(w)=2, \\ \frac{(x-1)(x-5)(x-9)}{120 x^{3}} & \text { if } d(w)=3 .\end{cases}
$$

This checks with $H_{H_{3}, x}$ (which as explained earlier is directly computable from tables in OS).

For the symmetric group, from [BaD] it emerges that $M_{S_{n}, x}(w)=\frac{\left(\begin{array}{c}x+n-1-d(w) \\ n\end{array}\right.}{x^{n}}$. To compute $H_{S_{n}, x}$, Proposition 2.1 of $\mathrm{OS}$ shows that $\chi\left(L^{z\left(w W_{K}\right)}, x\right)$ is equal to $(x-1) \cdots(x-(n-|K|)+1)$. Thus the face weight Definition 3 associates to $W_{K}$ is $\frac{\left(\begin{array}{c}x \\ n-|K|\end{array}\right)}{x^{n}}$, agreeing with the face weight for type $A$ shuffling in BHR]. For type $B$, Proposition 2.2 of $\left[\mathrm{OS}\right.$ shows that $\chi\left(L^{z\left(w W_{K}\right)}, x\right)$ is equal to $(x-1)(x-3) \cdots$ $(x-2(n-|K|)+1)$. Thus

$$
\begin{aligned}
H_{B_{n}, x}(w) & =\sum_{K \subseteq \Pi-\operatorname{Des}(w)} \frac{(-1)^{\operatorname{dim}\left(z\left(W_{K}\right)\right)} \chi\left(L^{z\left(w W_{K}\right)}, x\right)}{x^{n} \chi\left(L^{z\left(w W_{K}\right)},-1\right)} \\
& =\frac{1}{x^{n}} \sum_{j=0}^{n-d(w)}\left(\begin{array}{c}
n-d(w) \\
j
\end{array}\right)\left(\begin{array}{c}
\frac{x-1}{2} \\
n-j
\end{array}\right) \\
& =\frac{\left(\frac{x-1}{2}+n-d(w)\right.}{n},
\end{aligned}
$$

agreeing with the formula for $M_{B_{n}, x}$ in $[\mathrm{BB}$. The argument for type $C$ is identical.

Theorem 3 implies that the measures $H_{W, x}$ convolve when $W$ is of type $A, B, C$, $H_{3}$ or a rank 2 group. The measure $H_{W, x}$ does not always convolve. To see this, letting $w_{0}$ be the longest element one would have that

$$
H_{W,-x}(w)=H_{W,-1}\left(w_{0}\right) H_{W, x}\left(w_{0}^{-1} w\right)=H_{W, x}\left(w_{0}^{-1} w\right) .
$$

Since $\operatorname{Des}(w)=\Pi-\operatorname{Des}\left(w_{0}^{-1} w\right)$ this fails for many types (pages 156-157 of [F1]), and hence $H_{W, x}$ is not always equal to $M_{W, x}$.

\section{ACKNOWLEDGEMENTS}

The author thanks Persi Diaconis for keeping him well informed of his work, and a careful referee and reviewer for corrections. Part of this work was supported by an NSF Postdoctoral Fellowship.

\section{REFERENCES}

[BaD] Bayer, D. and Diaconis, P., Trailing the dovetail shuffle to its lair. Ann. of Appl. Probab. 2, Number 2 (1992), 294-313. MR 93d:60014

[BB] Bergeron, F. and Bergeron, N., Orthogonal idempotents in the descent algebra of $B_{n}$ and applications. J. Pure Appl. Algebra 79, Number 2 (1992), 109-129. MR 93f:20054 
[BBHT] Bergeron, F., Bergeron, N., Howlett, R.B., and Taylor, D.E., A decomposition of the descent algebra of a finite Coxeter group. J. Algebraic Combin. 1 (1992), 23-44. MR 93g:20079

[B3] Bergeron, F., and Bergeron, N., Symbolic manipulation for the study of the descent algebra of finite Coxeter groups. J. Symbolic Comput. 14 (1992), 127-139. MR 94d:68048

[B] Bidigare, P., Hyperplane arrangement face algebras and their associated Markov chains, Ph.D. Thesis, University of Michigan, 1997.

[BHR] Bidigare, P., Hanlon, P., and Rockmore, D., A combinatorial description of the spectrum of the Tsetlin library and its generalization to hyperplane arrangements, Duke Math. J. 99 (1999), 135-174. CMP 99:16

[BrD] Brown, K. and Diaconis, P., Random walk and hyperplane arrangements. Ann. of Probab. 26 (1998), 1813-1854. CMP 99:09

[C1] Carter, R., Finite groups of Lie type. John Wiley and Sons, 1985. MR 87d:20060

[C2] Carter, R., Conjugacy classes in the Weyl group. Composito Math. 25 (1972), 1-59. MR 47:6884

[F1] Fulman, J., Semisimple orbits of Lie algebras and card shuffling measures on Coxeter groups, J. Algebra 224 (2000), 151-165.

[F2] Fulman, J., Counting semisimple orbits of finite Lie algebras by genus, J. Algebra 217 (1999), 170-179. CMP 99:15

[F3] Fulman, J., The combinatorics of biased riffle shuffles. Ann. of Combin. 2 (1998), 1-6.

[F4] Fulman, J., Cellini's descent algebra, dynamical systems, and semisimple conjugacy classes of finite groups of Lie type, http://xxx.lanl.gov/abs/math.NT/9909121.

[F5] Fulman, J., Affine shuffles, shuffles with cuts, the Whitehouse module, and patience sorting, to appear in J. Algebra.

[Ha] Hanlon, P., The action of $S_{n}$ on the components of the Hodge decompositions of Hochschild homology, Michigan Math. J. 37 105-124. MR 91g:20013

[H] Humphreys, J., Reflection groups and Coxeter groups. Cambridge Studies in Advanced Mathematics 29, Cambridge University Press, Cambridge.

[OS] Orlik, P., and Solomon, L., Coxeter arrangements. Proc. Symposia in Pure Math. 40 (1983), Part 2, 269-291. MR 85b:32016

[SS] Shnider, S. and Sternberg, S., Quantum groups. Graduate Texts in Mathematical Physics, II. International Press, 1993. MR 95e:17022

[ST] Shephard, G.C., and Todd, J.A., Finite unitary reflection groups Canadian J. Math. 6 (1954), 274-304. MR 15:600b

[So1] Solomon, L., The orders of the finite Chevalley groups. J. Algebra 3 (1966), 376-393. MR 33:7424

[St] Stanley, R., Generalized riffle shuffles and quasi-symmetric functions, http://xxx.lanl.gov/abs/math.CO/9904042.

[Z] Zaslavsky, T., Facing up to arrangements: face-count formulas for partitions of space by hyperplanes. Mem. Amer. Math. Soc. 1 (154). MR 50:9603

Department of Mathematics, Dartmouth College, Hanover, New Hampshire 03755

E-mail address: fulman@dartmouth.edu

Current address: Department of Mathematics, Stanford University, Stanford, California 94305

E-mail address: fulman@math.stanford.edu 